\title{
ЗАКОНЫ НЬЮТОНА В НАГЛЯДНОЙ ИЛЛЮСТРАЦИИ ОПТИМИЗАЦИЯ УЧЕБНОГО ПРОЦЕССА
}

\author{
Ильина Татьяна Васильевна \\ преподаватель \\ ГАПОУ МО «Подмосковный колледж Энергия» \\ СП ЦСЛП «Энергия», г. Электроугли
}

Аннотация: График преподавания физики уплотнен, приходится искать новые пути, уложив прежний объём программы в меньшее количество часов, обеспечив необходимый фундамент знаний.

Во всех учебниках физики основные законы движения Ньютона изложены раздельно и не связаны между собой. Объединив их в одной иллюстрации, помогло мне выполнить эту задачу за один академический час.

Решение изобразить три закона Ньютона иллюстрацией дало возможность учащимся объёмно вникнуть в законы движения, применить математические знания для решения физических задач. Применить знания по информатике - создать таблицы, построить графики в программе Microsoft Excel. Попробовать свои силы в создании анимационного рисунка с помощью компьютерной проектной среды «Живая физика.

Ключевые слова: Три закона Ньютона в одной иллюстрации, рисунок 1. Второй закон Ньютона, рисунок 2. Третий закон Ньютона, рисунок 3. Первый закон Ньютона, рисунок 4.

Аналитический расчёт, таблица, рисунок 5. Графики скорости, ускорения и пройденного пути, рисунки 6, 7, 8.

\section{NEWTON'S LAWS IN A VISUAL ILLUSTRATION OPTIMIZATION OF THE EDUCATIONAL PROCESS}

\section{Ilyina Tatyana Vasilyevna}

Abstract: The schedule of teaching physics is compacted, we have to look for new ways, putting the previous volume of the program in a smaller number of hours, providing the necessary foundation of knowledge. 
In all physics textbooks, the basic laws of Newton's motion are set out separately and are not related to each other. Combining them in one illustration helped me to complete this task in one academic hour. The decision to depict Newton's three laws with an illustration made it possible for students to thoroughly understand the laws of motion, apply mathematical knowledge to solve physical problems. Apply your knowledge of computer science - create tables, build graphs in Microsoft Excel. Try your hand at creating an animated drawing using the computer project environment " Live Physics.

Key words: Three Newton's laws in one illustration, Figure 1. Newton's Second Law, Figure 2. Newton's Third Law, Figure 3. Newton's First Law, Figure 4.

Analytical calculation, table, Figure 5. Graphs of speed, acceleration and distance traveled, Figures 6, 7, 8 .

\section{ПЛАН-КОНСПЕКТ УРОКА}

TЕМА 2. Динамика

\section{ЗАКОНЫ ДВИЖЕНИЯ НЬЮТОНА}

УРОК № 5. Решение задач на применение законов Ньютона

ТИП УРОКА: комбинированный

ВИД УРОКА: традиционный в сочетании с программными педагогическимисредствами

ЦЕЛЬ УРОКА: передать три закона Ньютона в одной иллюстрации, дать возможность обучающимся наглядно вникнуть в законы движения. Привлечь внимание к изучению физического процесса на плакате, понять явление по своему сценарию. Научиться применять законы Ньютона к анализу явлений окружающего мира; применять знания на уроке к решению физических задач; усвоить характерные особенности законов.

\section{ЗАДАЧИ УРОКА:}

Образовательные: выявить уровень усвоения формул законов Ньютона и их применения. Повторить знания о параметрах, характеризующих массу, скорость, ускорении, силу. Почему тело движется равноускорено? Как возникает сила. При каких условиях тело движется без воздействия внешних сил. Что такое действие и противодействие.

Воспитательные: показать значение работ Ньютона, область их применения. Развития мышления: проверить уровень самостоятельного мышления, пробудить творческий поиск. 
СРЕДСТВА ОБУЧЕНИЯ: компьютер, мультимедийный проектор, иллюстрация Законы движения Ньютона.

\section{ХОД УРОКА:}

I. Иллюстрация.

Рисунок 1

1. «Рыцарь» копьём толкнул модель автомобиля массой один килограмм, в результате за одну секунду она сдвинулась на один метр, приобретя скорость

$\mathrm{V}_{1}=1 \mathrm{M} / \mathrm{c}$.

2. Определим ускорение $\mathrm{a}=V_{1}-V_{0} / t_{1}-t_{0}=1 \mathrm{M} / \mathrm{c}^{2}$.

3. Определим силу $\mathrm{F}$, необходимую для сдвига модели массой $\mathrm{m}=1$ кг за время $t=1 \mathrm{c}$. Она равна $1 \mathrm{H}$. $\boldsymbol{F}=\boldsymbol{m a}$ это второй закон Ньютона.

$\mathrm{F}=1 \kappa \Gamma \cdot 1 \mathrm{~m} / \mathrm{c}^{2}=1 \mathrm{H}$.

Эту силу назвали в честь Исаака Ньютона, рисунок 2

4. Модель с коробкой ударилась о кирпичную стену с силой $1 \mathrm{H}$. Это третий закон Ньютона - действие и противодействие

(модель автомобиля и неподвижная стена).

Модель автомобиля и стена действуют друг на друга с равными силами, но направленными в противоположные стороны

$$
\boldsymbol{F}=\boldsymbol{-} \boldsymbol{F} \text {, рисунок } 3
$$

5. На иллюстрации видно, что в момент столкновения коробка имела скорость 1 M $/ c$ и с этой же скоростью коробка продолжала двигаться по прямой линии до тех пор пока на неё не подействовали внешние силы. Это первый закон Ньютона - закон инерции, рисунок 4.

Все три закона не учитывают силы трения

II. Обучаемые, с целью понятия всех трёх законов Ньютона в одной иллюстрации интерпретируют её в своих тетрадях.

III. В тетрадях делают аналитический расчёт пройденного пути модели автомобиля массой 1 кг, при воздействии силы в один ньютон. Данные расчётов занося в сводную таблицу, рисунок 5. Строят графики зависимости скорости, ускорения и пройденного пути от времени $V=f(t), a=f(t), S=f(t)$, рисунок 6 графики.

Рекомендации: для построения графика $S=f(t)$.

1. Ось абсцисс (временная ось) в одну секунду. Делим на пять равных частей. 
2. Ускорение, $\mathrm{a}=$ const, $\mathrm{a}=1 \mathrm{~m} / \mathrm{c}^{2}$

3. По графику скорости $V=f(t)$ определяем скорости в каждый интервал времени методом интерполяции.

4. Определяем среднюю скорость

$\mathrm{V}_{\mathrm{cp} 1}=\left(\mathrm{V}_{0,2}-\mathrm{V}_{0}\right) / 2=0,2-0 / 2=0,1 \mathrm{~m} / \mathrm{cV}_{\mathrm{cp} 2}=\left(\mathrm{V}_{0,4}-\mathrm{V}_{0}\right) / 2=0,4-0 / 2=0,2$ $\mathrm{m} / \mathrm{cV}_{\mathrm{cp} 3}=\left(\mathrm{V}_{0,6}-\mathrm{V}_{0}\right) / 2=0,6-0 / 2=0,3 \mathrm{~m} / \mathrm{cV}_{\mathrm{cp} 4}=\left(\mathrm{V}_{0,8}-\mathrm{V}_{0}\right) / 2=0,8-0 / 2=0,4$ $\mathrm{m} / \mathrm{cV}_{\mathrm{cp} 5}=\left(\mathrm{V}_{1,0}-\mathrm{V}_{0}\right) / 2=0,2-0 / 2=0,5 \mathrm{~m} / \mathrm{c}$

5. Определяем пройденный путь $S_{1-5}$ за интервал времени $t=0,2 c$ по формуле: $\boldsymbol{S}=\boldsymbol{V}_{c p} \boldsymbol{t}+\boldsymbol{a} \boldsymbol{t}^{2} / \mathbf{2}$

IV. Домашнее задание. C помощью компьютера (или без него) и приложения Paint изобразить Законы движения Ньютона в одной иллюстрации по своему видению. Для желающих и имеющих программу «Живая физика» создать анимацию

\section{Список литературы}

1. Т.В. Ильина Интенсификация учебного процесса Законы движения Ньютона Теоретический и научно - методический журнал «Специалист» №3 Москва 2008

2. «Живая физика» Учебно - методический комлект Москва 2000.

\section{Приложения}

1. Рисунок 1 «Законы Ньютона в одной иллюстрации»

2. Рисунок 2 фрагмент слайда - «Второй закон Ньютона»

3. Рисунок 3 фрагмент слайда - «Третий закон Ньютона»

4. Рисунок 4 фрагмент слайда - «Первый закон Ньютона»

5. Рисунок 5 сводная таблица

6. Рисунки $6,7,8$ графики $V=f(t), a=f(t), S=f(t)$. 
13АКОН: при отсутствии внешиих сил, тело сохраняетсостояние покоя или равномерного двнжения без ускорения

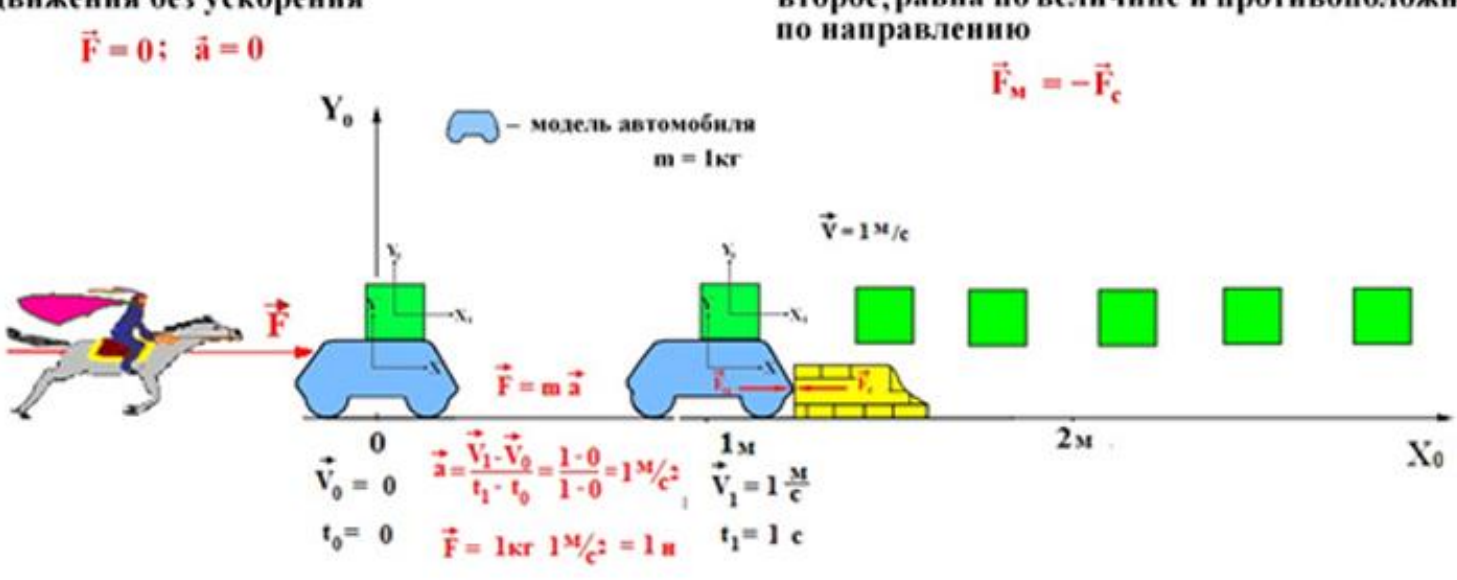

\section{2 ЗАКОН: сила, действующая на тело, равна произведению массы тела на его ускорение}

$$
\overrightarrow{\mathrm{F}}=\mathrm{m} \overrightarrow{\mathrm{a}}
$$

Рис. 1.

\section{Второй закон Ньютона}

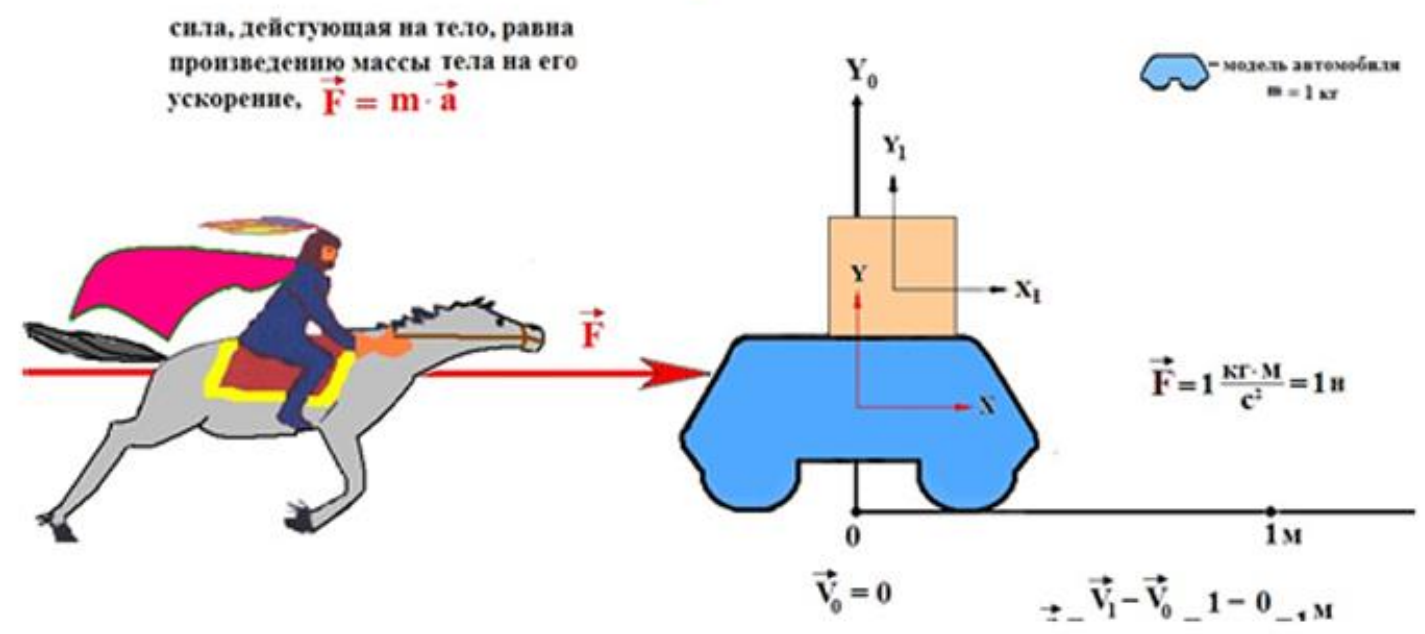

Рис. 2. 


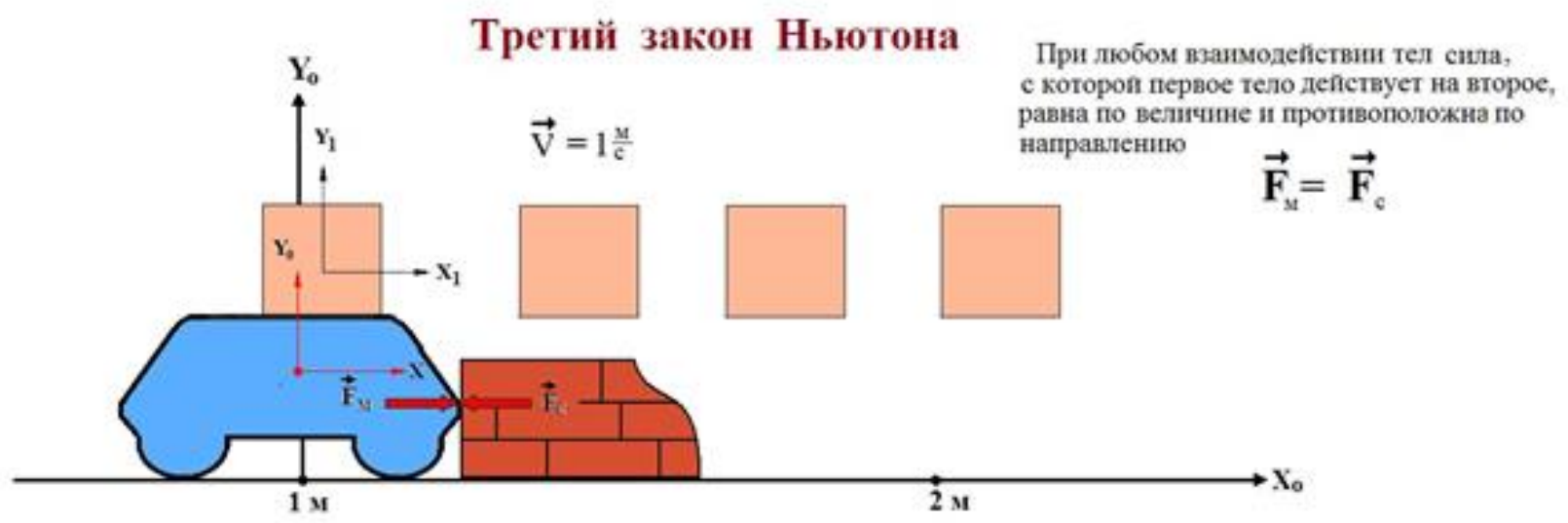

Рис. 3.

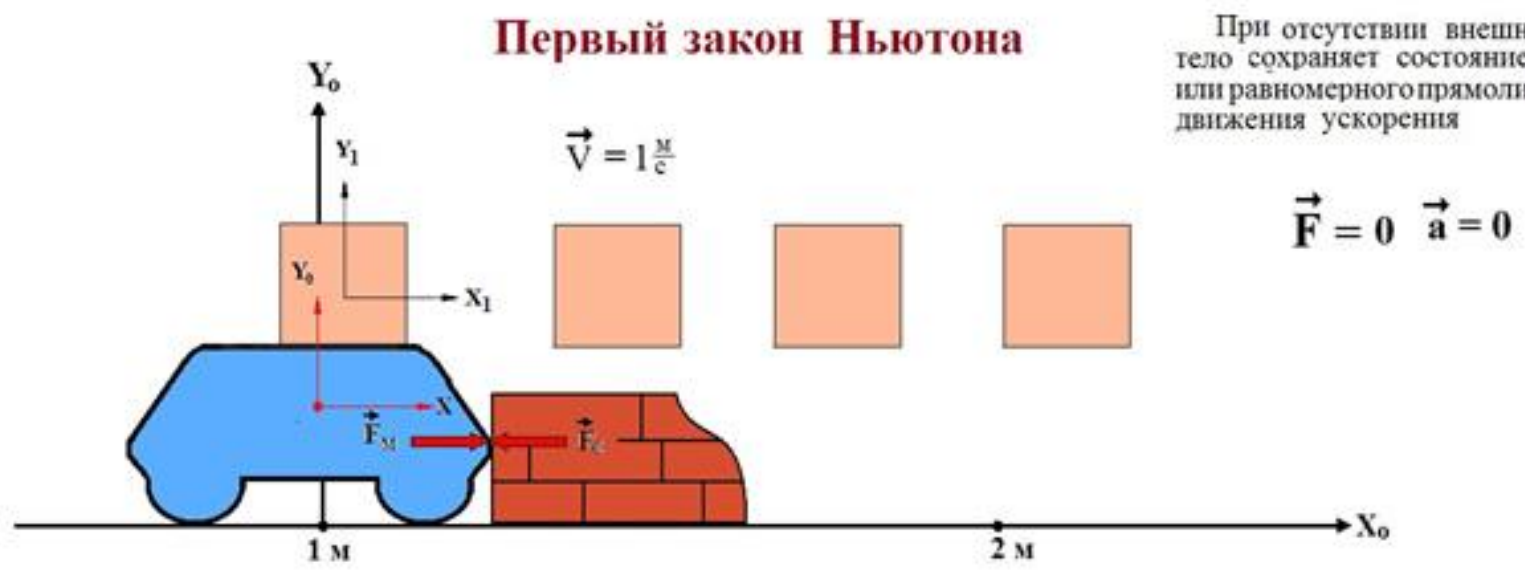

Рис. 4. 


\begin{tabular}{|c|c|c|c|c|}
\hline $\begin{array}{c}\text { Пройденный } \\
\text { путь } \\
S, M\end{array}$ & $\begin{array}{c}\text { Интервал } \\
\text { времени } \\
t_{2} c\end{array}$ & $\begin{array}{c}\text { Ускорение } \\
\qquad a, \frac{M}{c^{2}}\end{array}$ & $\begin{array}{c}\text { Средняя } \\
\text { Скорость } \\
V_{v p}, \frac{M}{n}\end{array}$ & $\begin{array}{l}\text { Расчёт по формуле } \\
S=V_{s p} \cdot t+\frac{a t^{2}}{2}, M\end{array}$ \\
\hline$S_{1}=0,04$ & 0,2 & 1 & 0,1 & $\begin{array}{l}S_{1}=0,1 \cdot 0,2+0,5.0,4= \\
S_{0,2} 0,02+0,02=0,04\end{array}$ \\
\hline$S_{2}=0,06$ & 0,4 & 1 & 0,2 & $\begin{array}{l}S_{2}=0,2 \cdot 0,4+0,5 \cdot 0,16= \\
S_{0,2} 0,02+0,02=0,04\end{array}$ \\
\hline$S_{3}=0,36$ & 0,6 & 1 & 0,3 & $\begin{array}{l}S_{3}=0,3 \cdot 0,6+0,5 \cdot 0,36= \\
S_{0,6} 0,18 \cdot 0,18=0,36\end{array}$ \\
\hline$S_{4}=0,64$ & 0,8 & 1 & 0,4 & $\begin{array}{l}S_{4}=0,4 \cdot 0,8+0,5 \cdot 0,64= \\
S_{0,8} 0,32 \cdot 0,32=0,64\end{array}$ \\
\hline$S_{5}=1,0$ & 1 & 1 & 0,5 & $\begin{array}{l}S_{5}=0,5 \cdot 1+0,5 \cdot 1,0= \\
S_{1} 0,5+0,5=1,0\end{array}$ \\
\hline
\end{tabular}

Рис. 5. Аналитический расчёт пройденного пути модели массой 1 кг при взаимодействии силыF = 1 ньютон

Графики. Скорости $V=f(t)$, пройденного пути $S=f(t)$, ускорения $a=f(t)$

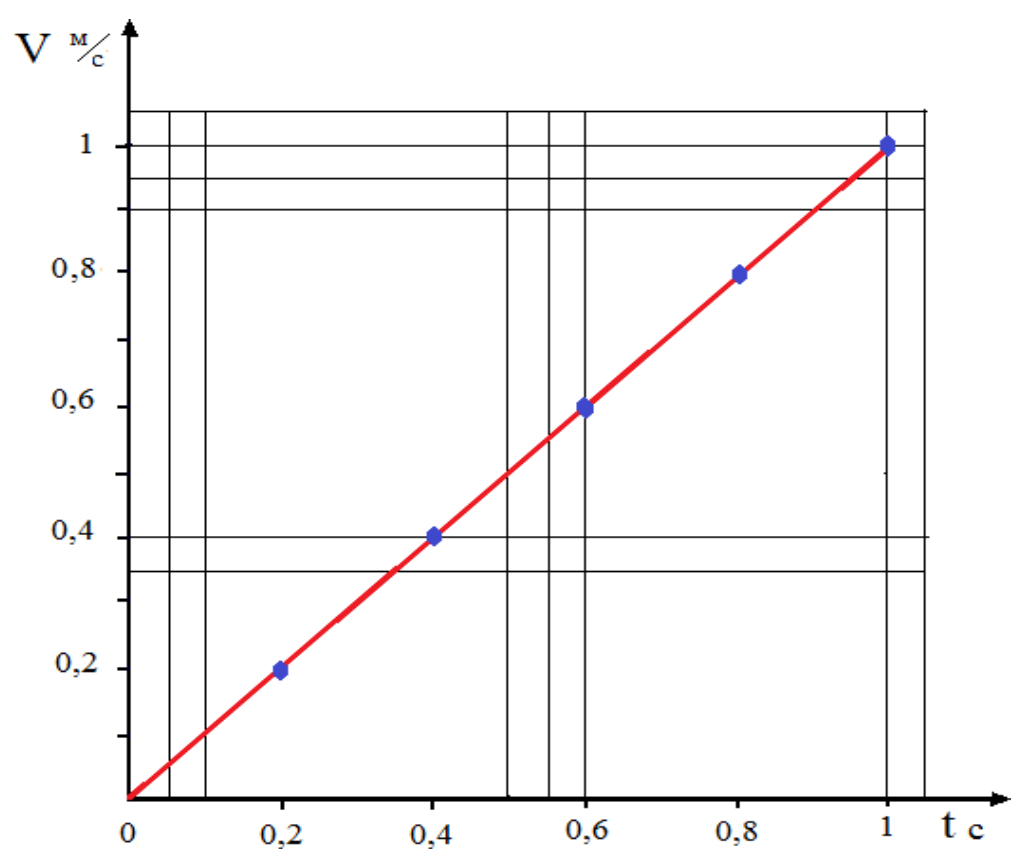

Рис. 6. 


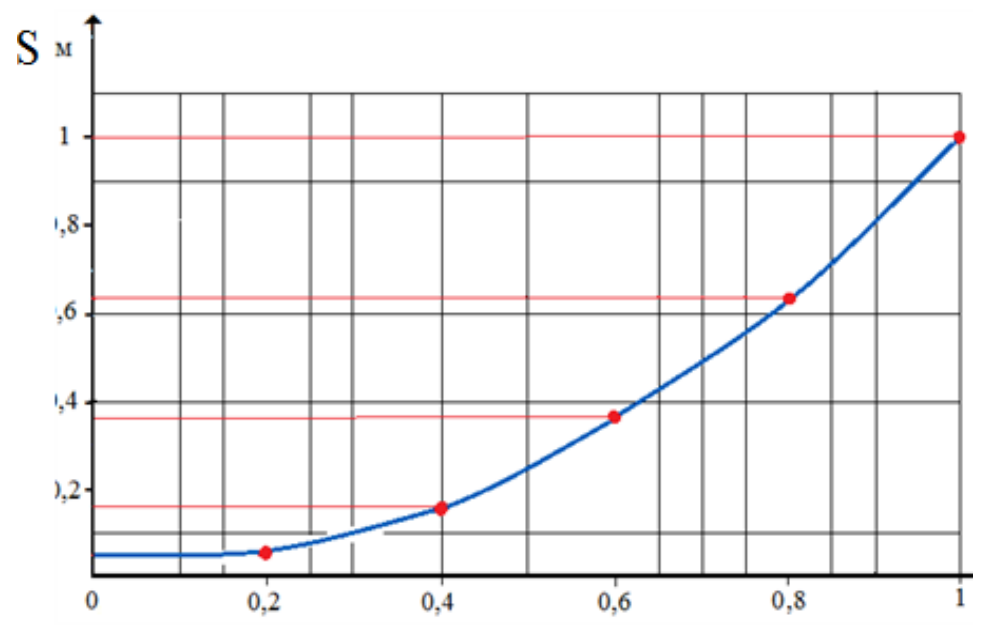

Рис. 7.

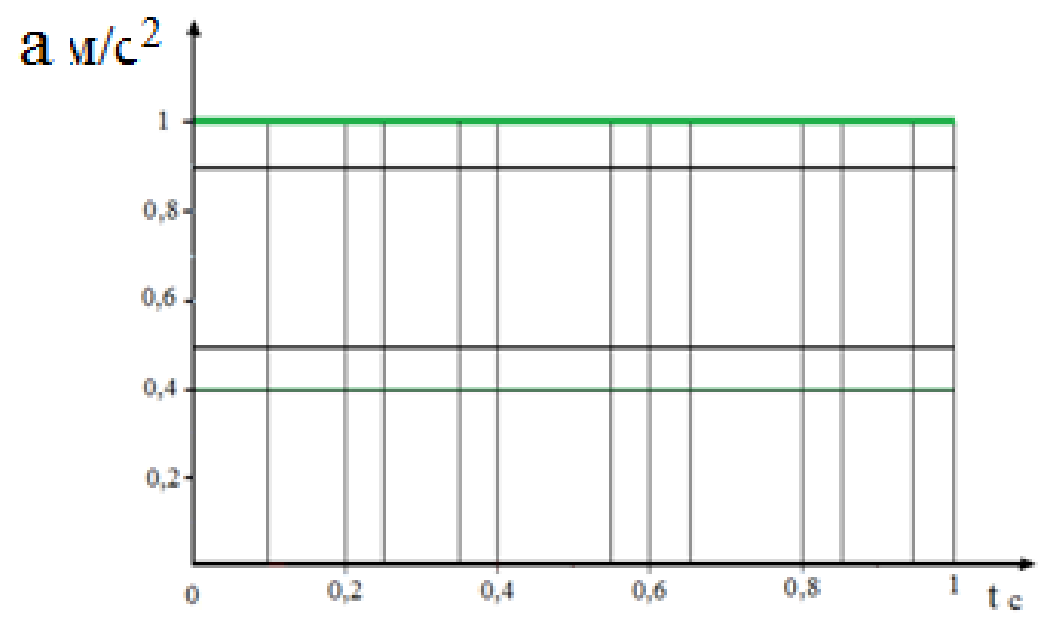

Рис. 8. 